\title{
Effects of CD44 and E-cadherin overexpression on the proliferation, adhesion and invasion of ovarian cancer cells
}

\author{
MEIYA MAO, XIAOJIAO ZHENG, BOHONG JIN, FUBIN ZHANG, LINYAN ZHU and LINING CUI \\ Department of Obstetrics and Gynecology, Ningbo First Hospital, Ningbo, Zhejiang 315035, P.R. China
}

Received November 16, 2016; Accepted June 29, 2017

DOI: $10.3892 /$ etm.2017.5259

\begin{abstract}
CD44 is a prognostic indicator of shorter survival time in ovarian cancer. E-cadherin fragmentation promotes the progression of ovarian cancer. However, the effects of CD44 and E-cadherin overexpression on ovarian cancer cells have remained elusive. The present study aimed to investigate the effects of overexpression of CD44 and E-cadherin on cell proliferation, adhesion and invasion of SKOV-3 and OVCAR-3 ovarian cancer cells. Overexpression of CD44 and E-cadherin was achieved by transfecting SKOV-3 and OVCAR-3 cells with viruses carrying the CD44 or E-cadherin gene, respectively. Expression of CD44 and E-cadherin was detected by western blot analysis. The proliferation of SKOV-3 and OVCAR-3 cells was measured by a Cell Counting Kit- 8 at 0, 24 and $48 \mathrm{~h}$ after viral transfection. The adhesion ability of SKOV-3 and OVCAR-3 cells to the endothelial layer was detected. A Transwell invasion assay was utilized to assess the invasion ability of the cells. Overexpression of CD44 and E-cadherin in SKOV-3 and OVCAR-3 cells was confirmed by western blot. Compared with the blank or negative control groups, the CD44 overexpression groups of SKOV-3 and OVCAR-3 cells exhibited an increased cell proliferation rate at 24 and $48 \mathrm{~h}$, whereas overexpression of E-cadherin did not alter the proliferation of these cells. Furthermore, compared with the blank and negative control groups, the cell adhesion and invasion ability in the CD44 overexpression groups of SKOV-3 and OVCAR-3 cells was markedly higher. There were no significant differences in adhesion ability between the E-cadherin overexpression group and the blank/negative control group. Of note, overexpression of E-cadherin decreased the invasive ability of SKOV-3 and OVCAR-3 cells. In conclusion, Overexpression of CD44 increased the proliferation, adhesion and invasion of ovarian cancer cells, while overexpression of E-cadherin decreased the invasion of ovarian cancer cells.
\end{abstract}

Correspondence to: Dr Lining Cui, Department of Obstetrics and Gynecology, Ningbo First Hospital, 59 Haishu Street, Ningbo, Zhejiang 315035, P.R. China

E-mail: 1935148739@qq.com

Key words: ovarian cancer, CD44, E-cadherin, proliferation, adhesion, invasion

\section{Introduction}

Ovarian cancer is a common malignant tumor type of the female reproductive system with a high mortality rate. The 5-year survival rate of patients with ovarian cancer is $25-30 \%$ (1). Although progress has been made in the treatment of ovarian cancer, the mortality rate remains high. The majority of ovarian cancer patients have tumor metastasis at the time-point of diagnosis, and tumor invasion and metastasis are important causes of treatment failure (2). Therefore, investigation of the molecular mechanisms involved in tumor proliferation, adhesion and invasion is of great importance for improving the therapeutic effectiveness of ovarian cancer treatments.

CD44 is a transmembrane protein that mediates cell-cell interactions as well as cell adhesion and migration. It is widely expressed on the cell surface (3). CD44 participates in numerous biological processes such as tumor metastasis, hematopoiesis, lymphocyte activation, recirculation and homing. CD44 is a receptor for hyaluronic acid and interacts with other ligands, such as matrix metalloproteinases, collagens and osteopontin (3). Positive CD44 expression was demonstrated to be a prognostic indicator of shorter survival time in patients with ovarian cancer (4-7). CD44 expression was also reported to contribute to the development of carboplatin resistance in advanced serous epithelial ovarian cancer, and contribute to a worse prognosis for these patients (7). In addition, epithelial cadherin (E-cadherin) is a calcium-dependent cell-cell adhesion glycoprotein. It interacts with the cytoskeleton, forming complexes with an anchoring effect. Genetic variants of the E-cadherin gene were reported to be a prognostic marker to identify patients at increased risk of invasive or metastatic cancer (8). E-cadherin fragmentation was revealed to promote the progression of intraperitoneal epithelial ovarian cancer (9). However, the effects of CD44 and E-cadherin overexpression on cell proliferation, adhesion and invasion of ovarian cancer cells have remained elusive.

Therefore, the present study aimed to investigate the effects of CD44 and E-cadherin overexpression on the proliferation, adhesion and invasion of SKOV-3 and OVCAR-3 ovarian cancer cells.

\section{Materials and methods}

Cells and reagents. The ovarian cancer cell lines SKOV-3 and OVCAR-3 were obtained from the Cell Bank of the Chinese 
Academy of Science (Shanghai, China). OVCAR-3 cells were cultured in RPMI-1640 (Thermo Fisher Scientific Inc., Waltham, MA, USA) supplemented with $20 \%$ fetal bovine serum (FBS; Thermo Fisher Scientific Inc.), $1 \%$ penicillin and streptomycin, and $10 \mu \mathrm{g} / \mathrm{ml}$ insulin (Beyotime Institute of Biotechnology, Shanghai, China). SKOV-3 cells were cultured in McCoy's 5A medium (Multicell Technologies Inc., Woonsocket, RI, USA) supplemented with $10 \%$ FBS. Cells were cultured at $37^{\circ} \mathrm{C}$ in a humidified atmosphere of $5 \% \mathrm{CO}_{2}$ and $95 \%$ air.

Reagents for vector construction and identification were as follows: Endonuclease EcoRI/BamHI, T4 DNA ligase, GeneRuler DNA ladder (Fermentas; Thermo Fisher Scientific Inc.); primer Oligo, 0.05\% Trypsin, Lipofectamine ${ }^{\circledR} 2000$ (Invitrogen; Thermo Fisher Scientific Inc.); gel extraction kit (AP-GX-50), plasmid extraction kit (AP-MN-P-50; Corning Inc., New York, NY, USA); packaging mix, 293T cells, cloning vector pcDNA3.1(+)/enhanced green fluorescence protein (EGFP)/internal ribosome entry site (IRES), lentiviral vector pL/GFP/IRES/MCS-BSD (Novobio Inc., Shanghai, China); Escherichia coli (E. coli) DH5a competent cells (Takara Biotechnology Inc., Dalian, China).

The following antibodies were used: Anti-GAPDH (1:5,000 dilution; bs-0755R, Biosscn Inc., Beijing, China); CD44 (1:1,000 dilution; bs-4916R), horseradish peroxidase-conjugated anti-rabbit immunoglobulin (Ig)G (1:5,000 dilution; bs-0295G-HRP), E-cadherin (1:1,000 dilution; bs-1519R).

Reagents used for cell proliferation, migration and adhesion assays were as follows: Bicinchoninic acid (BCA) kit (P0011, Beyotime Institute of Biotechnology); Cell Counting Kit-8 (CCK-8; CK04, Dojindo Molecular Technologies Inc., Rockville, MD, USA); Transwell plate ( $8 \mu \mathrm{m}$ pore width; Corning Inc.); crystal violet (Sigma-Aldrich; Merck KGaA, Darmstadt, Germany); $0.05 \%$ trypsin (Invitrogen; Thermo Fisher Scientific Inc.).

Construction of recombinant plasmids and lentiviral packaging. SKOV-3 and OVCAR-3 ovarian cancer cells were divided into 4 groups, respectively: Blank group, untransfected SKOV-3 or OVCAR-3 cells; negative control group, cells were transfected with empty viral vectors; CD44 overexpression group, cells were transfected with CD44 expression vector; and E-cadherin overexpression group, cells were transfected with E-cadherin expression vector.

The CD44 and E-cadherin genes were synthesized, individually cloned into pcDNA3.1(+)/EGFP/IRES cloning vector and amplified. The recombinant plasmids were extracted and cleaved by endonuclease. They were then inserted into the similarly digested lentiviral vector $\mathrm{pL} / \mathrm{GFP} / \mathrm{IRES} / \mathrm{MCS}-\mathrm{BSD}$ with T4 DNA ligase, and transformed into E. coli DH5a competent cells for amplification. The resulting recombinant lentiviral plasmids carrying CD44 and E-cadherin genes were named $\mathrm{pL} / \mathrm{to} / \mathrm{v} 5 / \mathrm{GFP} / \mathrm{IRES} / \mathrm{MCS}-\mathrm{V} 2-\mathrm{CD} 44$ and $\mathrm{pL} / \mathrm{to} / \mathrm{v} 5 / \mathrm{GFP} / \mathrm{IRES} / \mathrm{MCS}-\mathrm{V} 2-\mathrm{Ecad}$, respectively. Packaging mix $(9 \mu \mathrm{g})$ and recombinant lentiviral plasmids $(3 \mu \mathrm{g})$ were added into Opti-Minimum Essential Medium (MEM; $1.5 \mathrm{ml}$; Invitrogen; Thermo Fisher Scientific, Inc.) and mixed. Lipofectamine ${ }^{\circledR} 2000(36 \mu \mathrm{l})$ was mixed with Opti-MEM $(1.5 \mathrm{ml})$, and incubated at room temperature for $5 \mathrm{~min}$. The plasmid solution and diluted Lipofectamine ${ }^{\circledR} 2000$ were then mixed, and incubated at room temperature for $5 \mathrm{~min}$. The mixture was added into a culture dish with $293 \mathrm{~T}$ cells, and cells were cultured for $48 \mathrm{~h}$. Cell supernatant was then collected, centrifuged at $1,500 \mathrm{x}$ g for $10 \mathrm{~min}$ at room temperature and filtered. The virus solution was then condensed by centrifuging at $50,000 \mathrm{xg}$ for $2 \mathrm{~h}$ at $4^{\circ} \mathrm{C}$, and re-suspended in Dulbecco's modified Eagle's medium (Gibco; Thermo Fisher Scientific, Inc.).

Western blot analysis. Protein expression of CD44 and E-cadherin in SKOV-3 and OVCAR-3 ovarian cancer cells in the 4 groups was detected by western blot analysis. Cells were lysed in lysis buffer (P0013, Beyotime Institute of Biotechnology) with protease and phosphatase inhibitors (P1045, Beyotime Institute of Biotechnology) at $4^{\circ} \mathrm{C}$. The lysis mixture was centrifuged at $10,000 \mathrm{xg}$ for $10 \mathrm{~min}$ at $4^{\circ} \mathrm{C}$, and the supernatant containing cellular proteins was used in following experiments. The protein concentration was determined using a BCA kit. Proteins were separated by $10 \%$ SDS-PAGE (120 V) (40 $\mu \mathrm{g} / \mathrm{lane})$. The separated proteins were then transferred to polyvinylidene fluoride membranes (FFP24, Beyotime Institute of Biotechnology; $100 \mathrm{~V}$ for $120 \mathrm{~min})$. After being blocked with 5\% non-fat milk for $1 \mathrm{~h}$, the membranes were incubated with primary antibodies against CD44 and E-cadherin at $4^{\circ} \mathrm{C}$ overnight. Membranes were then washed with Tris-buffered saline containing Tween 20 and incubated with HRP-conjugated goat anti-rabbit secondary antibody at room temperature for $1 \mathrm{~h}$. Membranes were washed and incubated in enhanced chemiluminescence solution (P0018A, Beyotime Institute of Biotechnology), and images were captured on film (FF057, Beyotime Institute of Biotechnology) in a dark room. Experiments were repeated for 3 times.

Cell proliferation assay. The proliferation of SKOV-3 and OVCAR-3 ovarian cancer cells in the 4 groups was measured by a CCK- 8 cell proliferation assay kit at 0,24 and $48 \mathrm{~h}$ after ovarian cancer cells were transfected with the viruses. CCK- 8 solution was added to each well, followed by incubation for $4 \mathrm{~h}$. The absorbance was measured using a microplate reader at $490 \mathrm{~nm}$. Relative tumor cell proliferation rate was calculated by dividing the reading of each group at 24 or $48 \mathrm{~h}$ by the baseline reading at $0 \mathrm{~h}$. Experiments were repeated for 3 times.

Cell adhesion assay. Endothelial cells (2E4 cells; H-001F, HUVEC, Allcells, Alameda, CA, USA) were cultured in 96-well plates and grown as a monolayer, and SKOV-3 and OVCAR-3 cells $\left(2 \times 10^{4}\right.$ cells) from the 4 groups were individually added to the cell culture. SKOV-3 and OVCAR-3 cells were stained with Hoechst 33342 before the adhesion assay. Hoechst $33342(1 \mu \mathrm{g} / \mathrm{ml})$ was incubated with SKOV-3 and OVCAR-3 cells at room temperature for $10 \mathrm{~min}$, respectively. Cells were then washed with PBS twice. The cell mixture was then centrifuged at $1,000 \mathrm{xg}$ for $15 \mathrm{~min}$, and washed with PBS 3 times. The percentage of cells that adhered to the endothelial layer was evaluated under a microscope. The number of tumor cells remaining in each well was counted. The relative tumor cell adhesion ability was calculated by dividing the number of remaining tumor cells in each group by that in the blank group. Experiments were repeated for 3 times. 
A

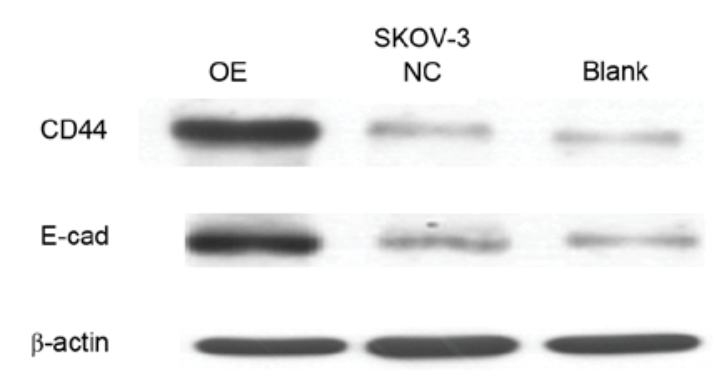

B

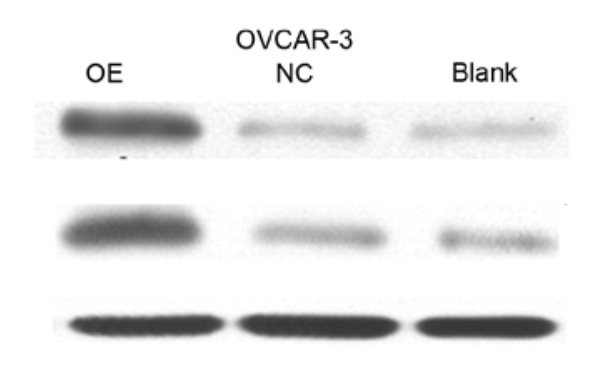

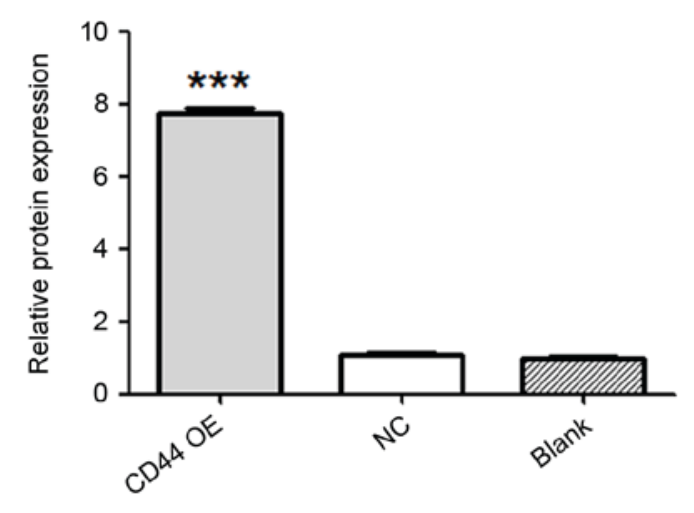
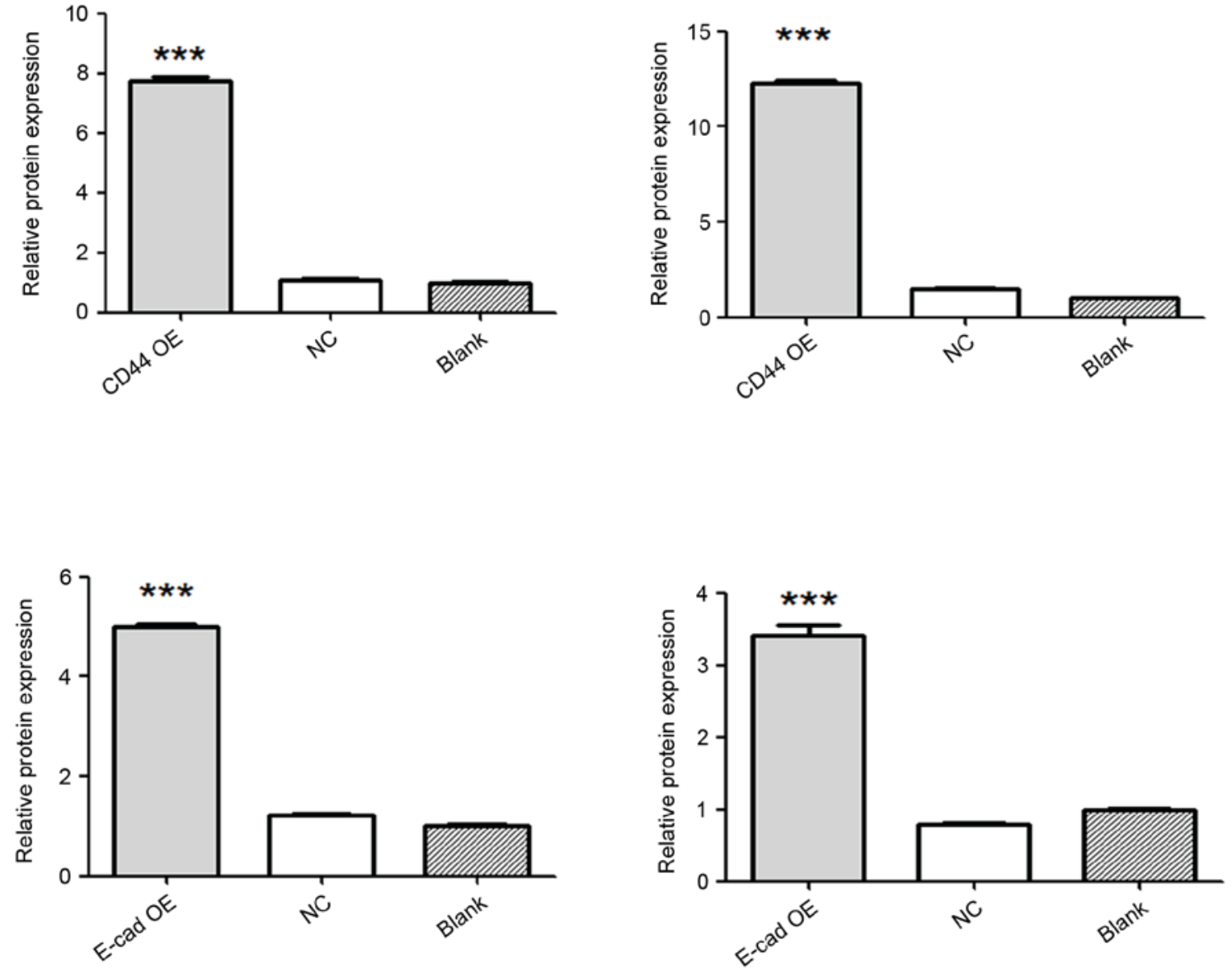

Figure 1. Confirmation of vector-mediated CD44 and E-cadherin overexpression by western blot analysis. Western blot image of protein expression of CD44 and E-cadherin in (A) SKOV-3 and (B) OVCAR-3 ovarian cancer cells. Relative protein expression of CD44 and E-cadherin is presented in the bar graphs below. Values are expressed as the mean \pm standard error of the mean $(\mathrm{n}=3 /$ group$) .{ }^{* * * *} \mathrm{P}<0.001$ vs. blank or negative control group. E-cad, epithelial cadherin; $\mathrm{NC}$, negative control; OE, overexpression.

Transwell invasion assay. The membrane of the upper compartment was coated with Matrigel (1 g/l; $50 \mu \mathrm{l})$, which was allowed to solidify by incubation at $37^{\circ} \mathrm{C}$ for $1 \mathrm{~h}$ SKOV-3 or OVCAR-3 cell suspension $\left(2 \times 10^{5}\right.$ cells $\left./ \mathrm{ml}, 200 \mu \mathrm{l}\right)$ was added to the upper compartment of each Transwell, while $800 \mu \mathrm{l}$ culture medium containing FBS was added to the lower compartment. Cells were incubated at $37^{\circ} \mathrm{C}$ for $24 \mathrm{~h}$. Subsequently, $4 \%$ paraformaldehyde was utilized to fix cells on the microporous membrane for $30 \mathrm{~min}$. Cells on the lower side of the membrane were stained with $1 \%$ crystal violet for $10 \mathrm{~min}$ and washed with PBS twice. Cells were then observed under a microscope (magnification, $\mathrm{x} 400$ ), and the number of cells that had transgressed through the membrane was counted. Relative tumor cell invasion was calculated by dividing the average number of cells that invaded through the membrane in the experimental groups by that in the blank group. Experiments were repeated for 3 times.

Statistical analysis. The statistical data were analyzed by GraphPad Prism 5.0 software (GraphPad Software Inc., La Jolla, CA, USA). Values are expressed as the mean \pm standard error of the mean. Differences among 3 or more groups were compared by analysis of variance, followed by the Bonferroni post-hoc test for multiple comparisons. 
A

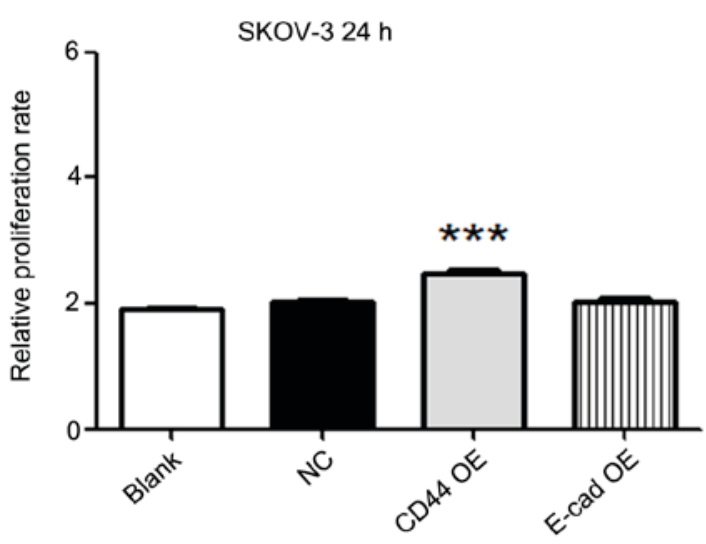

C

OVCAR-3 $24 \mathrm{~h}$

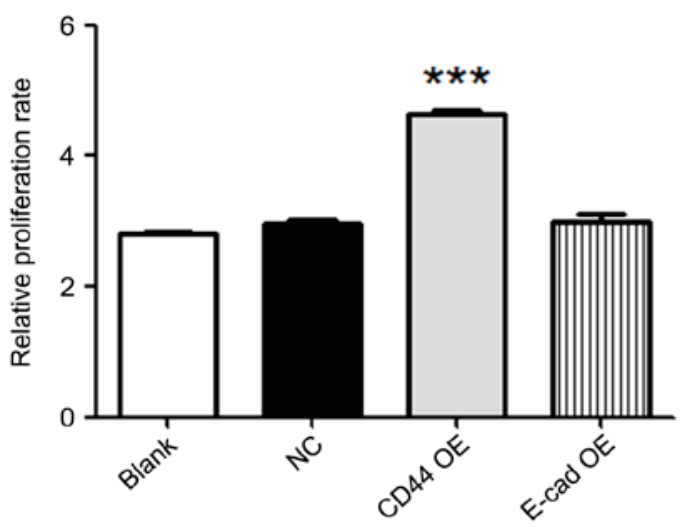

B

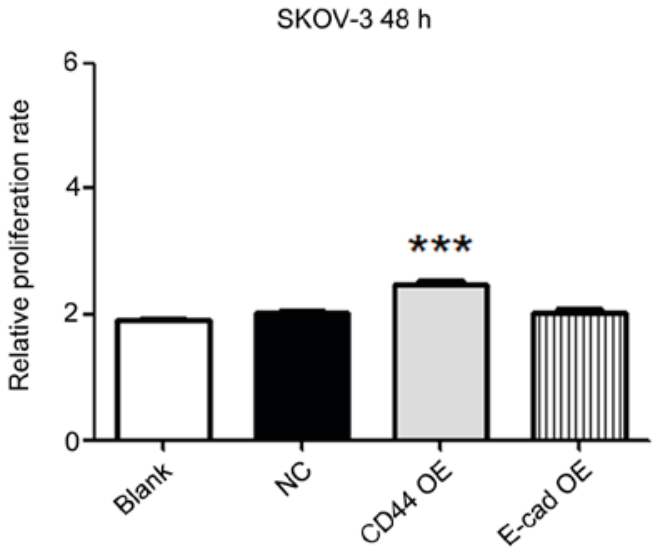

D

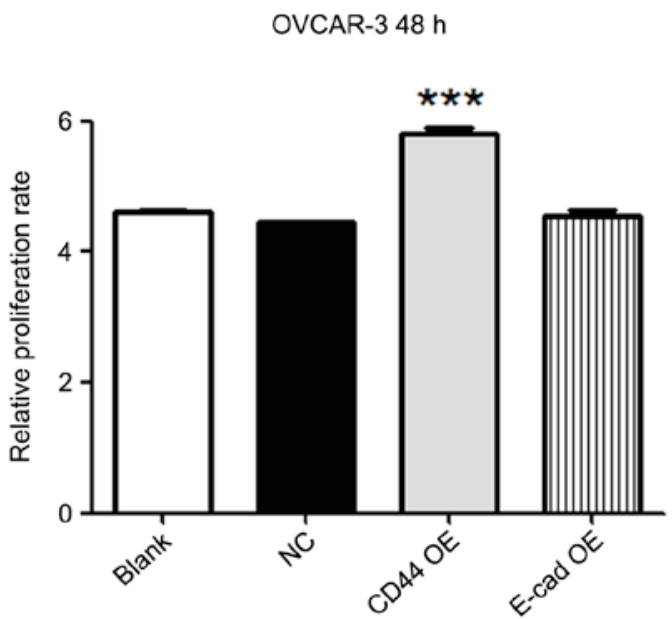

Figure 2. Overexpression of CD44 increases the proliferation of ovarian cancer cells. The proliferation of SKOV-3 cells at (A) $24 \mathrm{~h}$ and (B) $48 \mathrm{~h}$; and OVCAR-3 ovarian cancer cells at (C) $24 \mathrm{~h}$ and (D) $48 \mathrm{~h}$ in 4 groups was measured by a Cell Counting Kit-8 at 0,24 and $48 \mathrm{~h}$ after viral transfection. The relative tumor cell proliferation rate was calculated by dividing the reading of each group at 24 or $48 \mathrm{~h}$ by the baseline reading at $0 \mathrm{~h}$, respectively. Compared with the blank or negative control groups, the CD44 overexpression groups of SKOV-3 and OVCAR-3 cells had an increased proliferation rate at 24 and 48 h. However, overexpression of E-cadherin did not alter the proliferation of SKOV-3 and OVCAR-3 cells. Values are expressed as the mean \pm standard error of the mean (n=3/group). ${ }^{* * *} \mathrm{P}<0.001$ vs. blank or negative control group. E-cad, epithelial cadherin; NC, negative control; OE, overexpression.

$\mathrm{P} \leq 0.05$ was considered to indicate a statistically significant difference.

\section{Results}

Confirmation of $\mathrm{CD} 44$ and E-cadherin overexpression by western blot. SKOV-3 and OVCAR-3 ovarian cancer cells were divided into 4 groups as described above. Overexpression of CD44 and E-cadherin was achieved by transfecting SKOV-3 and OVCAR-3 cells with viruses carrying the CD44 or E-cadherin gene. Expression of CD44 and E-cadherin was detected by western blot analysis. Compared with that in the negative control and blank groups, the protein expression of CD44 in the CD44 overexpression group was significantly increased $(\mathrm{P}<0.001)$, and the protein expression of E-cadherin markedly increased in the E-cadherin overexpression group $(\mathrm{P}<0.001$; Fig. 1).

Overexpression of CD44 increases the proliferation of ovarian cancer cells. The proliferation of SKOV-3 and
OVCAR-3 ovarian cancer cells in the 4 groups was determined by a CCK- 8 cell proliferation assay at 0,24 and $48 \mathrm{~h}$ after viral transfection. The results demonstrated that compared with the blank or negative control group, the CD44 overexpression group had an increased cell proliferation rate at 24 and $48 \mathrm{~h}$ in SKOV-3 $(\mathrm{P}<0.001)$ as well as in OVCAR-3 cells ( $\mathrm{P}<0.001$; Fig. 2). However, overexpression of E-cadherin did not alter the proliferation of SKOV-3 and OVCAR-3 cells as compared with that in the blank or negative control groups.

CD44 overexpression increases the adhesion ability of ovarian cancer cells. The adhesion ability of SKOV-3 and OVCAR-3 cells to an endothelial layer was detected. Compared with that in the blank and negative control groups, the adhesion ability in the CD44 overexpression group was significantly higher in SKOV-3 as well as OVCAR-3 cells $(\mathrm{P}<0.001$; Fig. 3$)$. There were no significant differences in adhesion ability between the E-cadherin overexpression groups and the blank/negative control groups. 
A

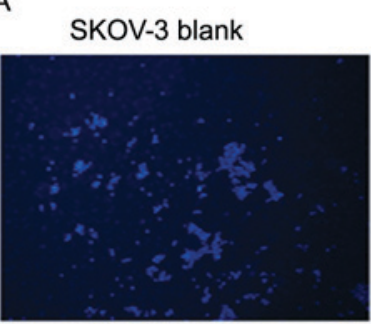

OVCAR-3 blank

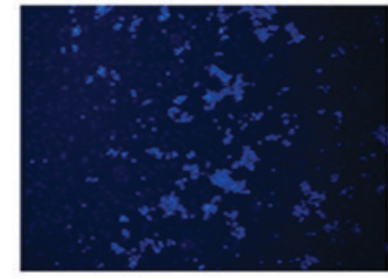

B

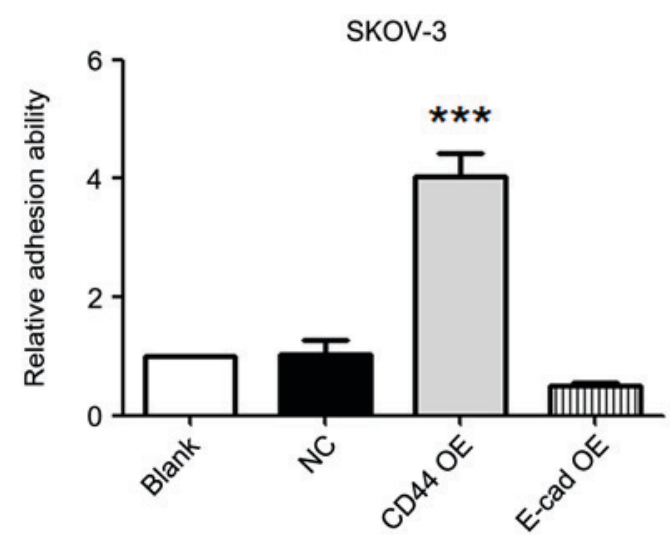

CD44 OE

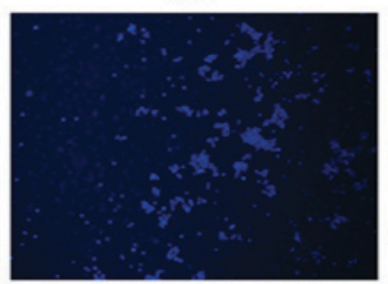

NC
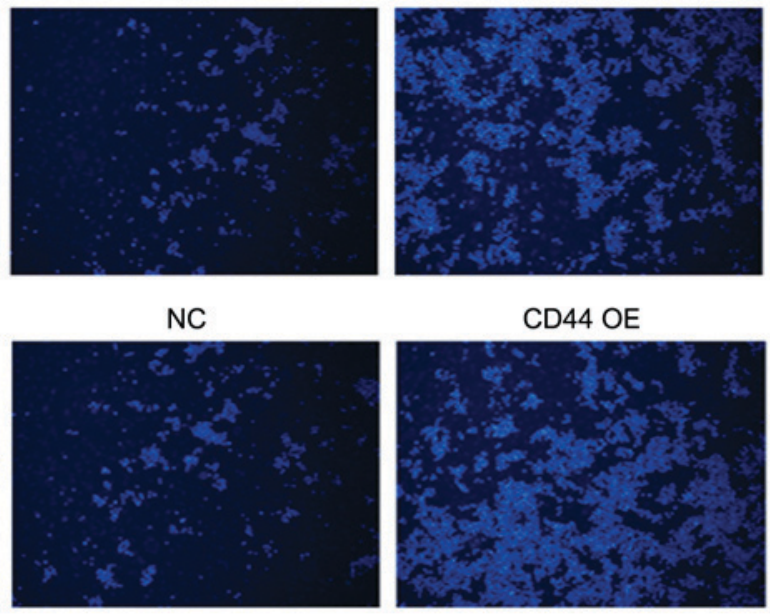

CD44 OE

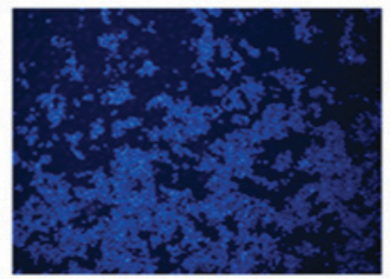

E-cad OE

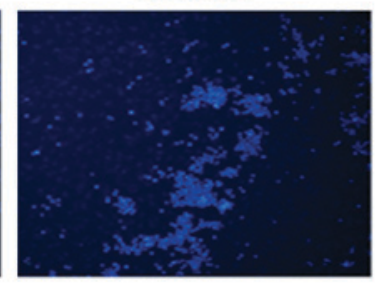

E-cad OE

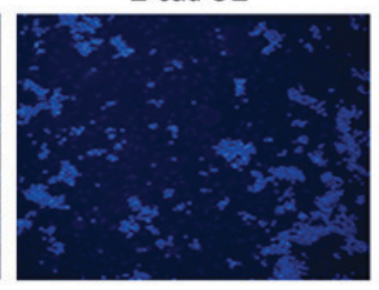

C

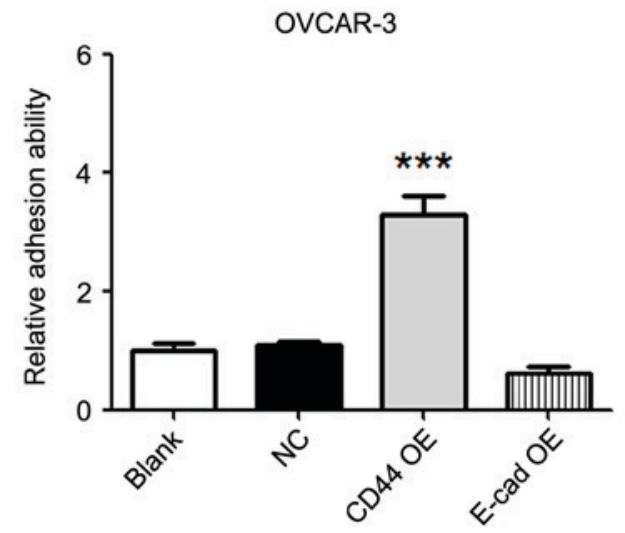

Figure 3. CD44 overexpression increases the adhesion ability of ovarian cancer cells. (A) Images presenting the cells adhering to the endothelial layer in each group (magnification, x40; Hoechst 33342 stain). (B and C) Relative adhesion ability of SKOV-3 and OVCAR-3 cells. Compared with that in the blank and negative control groups, the adhesion ability of SKOV-3 and OVCAR-3 cells in the CD44 overexpression group was markedly higher. There were no significant differences in adhesion ability between the E-cadherin overexpression group and the blank/negative control group. Values are expressed as the mean \pm standard error of the mean ( $\mathrm{n}=3$ /group). ${ }^{* * *} \mathrm{P}<0.001$ vs. blank or negative control group. E-cad, epithelial cadherin; NC, negative control; OE, overexpression.

Effects of overexpression of CD44 and E-cadherin on invasion of ovarian cancer cells. A Transwell invasion assay was utilized to detect the tumor invasion ability. Compared with that in the blank or negative control groups, overexpression of CD44 in SKOV-3 and OVCAR-3 cells increased the tumor invasion ability $(\mathrm{P}<0.001$; Fig. 4$)$. In addition, overexpression of E-cadherin in SKOV-3 and OVCAR-3 cells decreased the tumor invasion ability compared with that in the blank or negative control groups $(\mathrm{P}<0.001$; Fig. 4).

\section{Discussion}

The present study demonstrated that overexpression of CD44 increased the proliferation, adhesion and invasion of ovarian cancer cells. Overexpression of E-cadherin only decreased the invasion of ovarian cancer cells.

A large number of studies have shown that proliferation, adhesion, invasion and metastasis are the most important biological behaviors of malignant tumors. Tumor cells from the original site invade into surrounding tissue, penetrate the vascular basement membrane or lymph duct, and seed into distal organs via the blood or lymph circulation (10). During invasion and metastasis, tumor cells interact with the extracellular matrix and host cells via adhesion molecules. Alteration of adhesion molecules may change the proliferation, adhesion and invasion of tumor cells $(11,12)$.

CD44 was reported to be involved in the epithelial-to-mesenchymal transition of ovarian cancer cells (13). Furthermore, the CD44 splice variant v8-10 was reported to be a marker of serous ovarian cancer prognosis (6). An ovarian cancer tissue microarray analysis of paired primary, metastatic and recurrent tumor tissues from 26 patients, demonstrated that the metastatic as well as recurrent ovarian cancer tissues expressed higher levels of CD44 than the primary tumor (14). In addition, a meta-analysis unveiled that patients with CD44 was associated with poor prognosis in ovarian cancer patients, and that a $\mathrm{CD} 44^{+}$status was associated with common clinicopathological features and poor prognostic factors (5).

In line with the abovementioned studies, the present study also revealed that overexpression of CD44 increased the proliferation, adhesion and invasion of ovarian cancer cells. Increased interaction of osteopontin with CD44 was reported to be correlated with increased mitosis and in vitro 
A

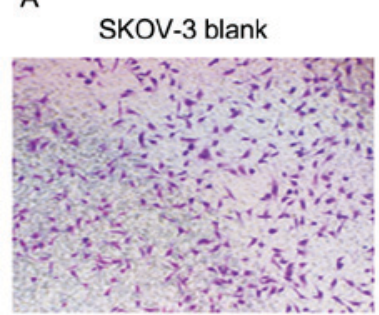

OVCAR-3 blank

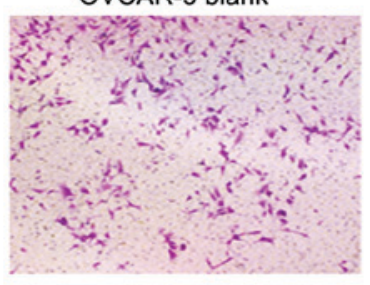

B

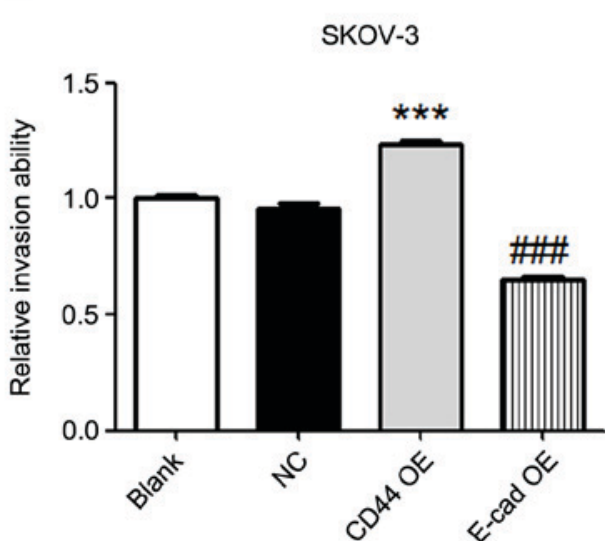

NC

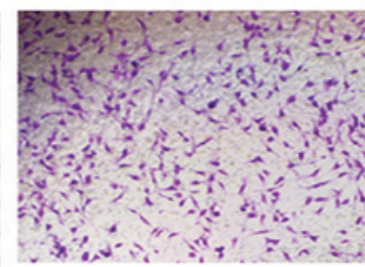

NC

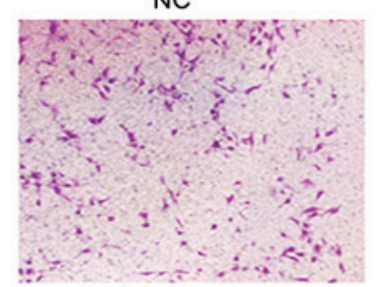

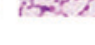

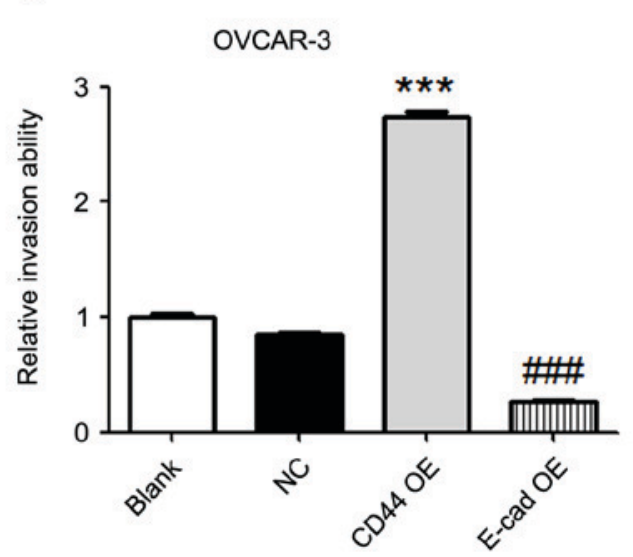

Figure 4. Effects of CD44 and E-cadherin overexpression on the invasion of ovarian cancer cells. (A) Image of cells that had transgressed through the membrane in a Transwell invasion assay (magnification, $\mathrm{x} 40$; crystal violet stain). (B and C) Relative invasion ability of SKOV-3 and OVCAR-3 cells. Overexpression of CD44 in SKOV-3 and OVCAR-3 cells increased the tumor invasion ability compared with that in the blank or negative control groups. In addition, overexpression of E-cadherin in SKOV-3 and OVCAR-3 cells decreased the tumor invasion ability compared with that in the blank or negative control groups. Values are expressed as the mean \pm standard error of the mean (n=3/group). ${ }^{* * * *} \mathrm{P}<0.001$ vs. blank or negative control group; ${ }^{\# \# \# ~} \mathrm{P}<0.001$ vs. blank or negative control group. E-cad, epithelial cadherin; $\mathrm{NC}$, negative control; OE, overexpression.

proliferation-enhancing effects in gastrointestinal stromal tumor cells (15). M2 macrophages-secreted osteoactivin/glycoprotein non-metastatic melanoma protein B was reported to enhance the viability, proliferation and migration of mesenchymal stem cells through extracellular signal-regulated kinase (ERK) and AKT signaling pathways via CD44 (16). In addition, biological changes induced by CD44 silencing were revealed to be mediated by cumulative downregulation of c-Jun, Sp1 and c-Src in human breast cancer cells (17). The molecular mechanisms underlying the effects of CD44 overexpression on ovarian cancer cells remain to be fully clarified. Whether the altered proliferation, adhesion and invasion ability of ovarian cancer cells are associated with osteopontin, the ERK and AKT signaling pathway, c-Jun, Sp1 and c-Src requires further investigation.

In addition, the present study demonstrated that overexpression of E-cadherin decreased the invasion of ovarian cancer cells. A previous study unveiled that E-cadherin expression was a predictor of better responses to first-line platinum-based chemotherapy, platinum sensitivity and favorable clinical outcome in patients with advanced-stage ovarian cancer (18). Furthermore, transforming growth factor a was revealed to induce the invasion of human ovarian cancer cells by downregulating E-cadherin (19). Betacellulin induced ovarian cancer migration and Slug-dependent downregulation of E-cadherin via epidermal growth factor receptor-mediated mitogen-activated protein kinase kinase/ERK and phosphoinositide-3 kinase/Akt signaling (20). Further research efforts are required to elucidate the key molecular pathways triggered by E-cadherin overexpression.

In conclusion, the present study highlighted the crucial role of CD44 in promoting the proliferation, adhesion and invasion of ovarian cancer cells. Overexpression of E-cadherin decreased the invasion of ovarian cancer cells. Although further studies on the underlying molecular mechanisms are required, the present results may provide a foundation for possible pharmaceutical targeting of ovarian cancer.

\section{Acknowledgements}

This study was funded by the Natural Science Foundation of Ningbo (grant nos. 2013A610225, 2013A610228 and 2015A610228) and the Medical and Health Plan of Zhengjiang (grant no. 2015KYA198). 


\section{References}

1. Auersperg N, Wong AS, Choi KC, Kang SK and Leung PC: Ovarian surface epithelium: Biology, endocrinology, and pathology. Endocr Rev 22: 255-288, 2001.

2. Siegel R, Naishadham D and Jemal A: Cancer statistics, 2013. CA Cancer J Clin 63: 11-30, 2013.

3. Jia Q, Feng M, Wang Y and Xue S: Gastric cancer cells in collagen gel matrix: Three-dimensional growth and differential expression of adhesion molecules (CD44s, CD54, E-cadherin). J Biomed Mater Res A 84: 917-925, 2008.

4. Karan Križanac D, Krasić Arapović A, Skočibušić S, Pintarić I, Trgo G and Tomić S: CD44 Immunoexpression is unfavorable predictor in ovarian serous cancer. Appl Immunohistochem Mol Morphol: Aug 3, 2016 (Epub ahead of print).

5. Shi YY and Jiang H: Prognostic role of the cancer stem cell marker CD44 in ovarian cancer: A meta-analysis. Genet Mol Res: doi: $10.4238 / \mathrm{gmr} .15038325$

6. Sosulski A, Horn H, Zhang L, Coletti C, Vathipadiekal V, Castro CM, Birrer MJ, Nagano O, Saya H, Lage K, et al: CD44 Splice Variant v8-10 as a marker of serous ovarian cancer prognosis. PLoS One 11: e0156595, 2016.

7. Elzarkaa AA, Sabaa BE, Abdelkhalik D, Mansour H, Melis M, Shaalan W, Farouk M, Malik E and Soliman AA: Clinical relevance of CD44 surface expression in advanced stage serous epithelial ovarian cancer: A prospective study. J Cancer Res Clin Oncol 142: 949-958, 2016.

8. Juan W, Shan K, Na W, Rong-Miao Z and Yan L: The associations of genetic variants in E-cadherin gene with clinical outcome of epithelial ovarian cancer. Int J Gynecol Cancer 26: 1601-1607, 2016.

9. Trillsch F, Kuerti S, Eulenburg C, Burandt E, Woelber L, Prieske K, Eylmann K, Oliveira-Ferrer L, Milde-Langosch K and Mahner S: E-Cadherin fragments as potential mediators for peritoneal metastasis in advanced epithelial ovarian cancer. $\mathrm{Br} J$ Cancer 114: 213-220, 2016.

10. Henic E, Noskova V, Høyer-Hansen G, Hansson S and Casslén B: Estradiol attenuates EGF-induced rapid uPAR mobilization and cell migration via the G-protein-coupled receptor 30 in ovarian cancer cells. Int J Gynecol Cancer 19: 214-222, 2009.
11. Banerjee S and Kaye SB: New strategies in the treatment of ovarian cancer: Current clinical perspectives and future potential. Clin Cancer Res 19: 961-968, 2013.

12. Lengyel E: Ovarian cancer development and metastasis. Am J Pathol 177: 1053-1064, 2010.

13. Vos MC, Hollemans E, Ezendam N, Feijen H, Boll D, Pijlman B, van der Putten $H$, Klinkhamer $P$, van Kuppevelt TH, van der Wurff AA and Massuger LF: MMP-14 and CD44 in Epithelial-to-Mesenchymal Transition (EMT) in ovarian cancer. J Ovarian Res 9: 53, 2016.

14. Gao Y, Foster R, Yang X, Feng Y, Shen JK, Mankin HJ, Hornicek FJ, Amiji MM and Duan Z: Up-regulation of CD44 in the development of metastasis, recurrence and drug resistance of ovarian cancer. Oncotarget 6: 9313-9326, 2015.

15. Hsu KH, Tsai HW, Lin PW, Hsu YS, Shan YS and Lu PJ: Osteopontin expression is an independent adverse prognostic factor in resectable gastrointestinal stromal tumor and its interaction with CD44 promotes tumor proliferation. Ann Surg Oncol 17: 3043-3052, 2010.

16. Yu B, Sondag GR, Malcuit C, Kim MH and Safadi FF: Macrophage-Associated Osteoactivin/GPNMB Mediates Mesenchymal stem cell survival, proliferation, and migration via a CD44-dependent mechanism. J Cell Biochem 117: 1511-1521, 2016.

17. Nam K, Oh S, Lee KM, Yoo SA and Shin I: CD44 regulates cell proliferation, migration, and invasion via modulation of c-Src transcription in human breast cancer cells. Cell Signal 27: 1882-1894, 2015.

18. Miše BP, Telesmanić VD, Tomić S, Šundov D, Čapkun V and Vrdoljak E: Correlation between E-cadherin Immunoexpression and efficacy of first line platinum-based chemotherapy in advanced high grade serous ovarian cancer. Pathol Oncol Res 21: 347-356, 2015.

19. Qiu X, Cheng JC, Klausen C, Fan Q, Chang HM, So WK and Leung PC: Transforming growth factor- $\alpha$ induces human ovarian cancer cell invasion by down-regulating E-cadherin in a Snail-independent manner. Biochem Biophys Res Commun 461: $128-135,2015$.

20. Zhao J, Klausen C, Qiu X, Cheng JC, Chang HM and Leung PC: Betacellulin induces Slug-mediated down-regulation of E-cadherin and cell migration in ovarian cancer cells. Oncotarget 7: 28881-28890, 2016. 